\title{
Process Study on Surface Modification of Coral Hydroxyapatite
}

\author{
Chang Jiahe ${ }^{1}$, Su Jun ${ }^{2}$, Zhang Wenyun ${ }^{2, *}$ \\ ${ }^{1}$ Heping Hospital Affiliated to Changzhi Medical College, Changzhi, China \\ ${ }^{2}$ Department of Stomatology, No. 920 Hospital of PLA, Kunming Medical University, Kunming, China \\ Email address: \\ 383778677@qq.com (Chang Jiahe), wenyunzh88@126.com (Zhang Wenyun) \\ ${ }^{*}$ Corresponding author
}

To cite this article:

Chang Jiahe, Su Jun, Zhang Wenyun. Process Study on Surface Modification of Coral Hydroxyapatite. American Journal of Clinical and Experimental Medicine. Vol. 9, No. 5, 2021, pp. 151-156. doi: 10.11648/j.ajcem.20210905.15

Received: September 9, 2021; Accepted: October 4, 2021; Published: October 12, 2021

\begin{abstract}
Objective: To explore process of modifying coral hydroxyapatite by $\mathrm{nmZnO}$ under different conditions, the final plan is to develop a porous artificial bone composite that combines the antibacterial properties of nano zinc oxide with the porous biodegradability of coral hydroxyapatite. Methods: Coral hydroxyapatite was modified by zinc nitrate sol-gel method at $70^{\circ} \mathrm{C}$ in weak acid environment. White granular porous composite materials were obtained by ultrasonic, rotary stirring, drying and calcination. The composition of the composite material is analyzed using X-ray diffractomer (XRD), using scanning electron microscopy (SEM) to observe and analyze changes in the surface appearance of composite materials, using energy dispersive X-ray spectroscopy (EDX) to observe and analyze the composition of the composite surface, the results of thermogravimetric analysis were used to study the decomposition temperature and other characteristics of the composite. Results: The sol-gel method can be used for antibacterial modification on CHA surface. When the mass ratio of coral hydroxyapatite, zinc nitrate and PEG-6000 is 48:4:5, the particle size and distribution of nano-zinc oxide particles are ideal, and uniformly distributed spherical $\mathrm{ZnO}$ nanoparticles can be observed under scanning electron microscopy. Conclusion: Coral hydroxyapatite surface could be modified by zinc nitrate sol-gel method. The particle size of nano zinc oxide is less than 100 nanometers. The agglomeration problem of nano-particles is solved; the porous structure of CHA are not destroyed.
\end{abstract}

Keywords: Coral Hydroxyapatite, Zinc Oxide, Modification, Antibacterial Property

\section{Introduction}

Oral and maxillofacial fractures and bone tumors caused by trauma and poor living habits have become increasingly common in recent years [1-4]. This can cause severe bone defect problems, causing physical dysfunction and mental trauma. The bone graft materials currently used in clinical work usually do not have antimicrobial properties. The incidence of infection after bone grafting is high and can easily lead to failure of bone grafting. Therefore, it is necessary to develop a porous bone repair material that combines good biocompatibility, osteogenic activity, long-lasting broad-spectrum antimicrobial properties and does not develop drug resistance.

In 1974, Roy [5] adopted natural coral to undergo hydrothermal exchange reaction, which made coral change calcium carbonate into hydroxyapatite while keeping the original porosity unchanged, thus reducing the degradation rate of the material. Coral hydroxyapatite was applied to repair bone defects. Coral hydroxyapatite artificial bone is considered as an ideal bone substitute material, and because of its good biocompatibility and natural three-dimensional porous structure, it is also considered as a promising scaffold material and carrier for bone tissue engineering [6-7].

Among the antimicrobial biomaterials, $\mathrm{ZnO}$ nanoparticles are abundant in raw material resources, less expensive, non-toxic to the environment, and have wide band gaps, UV absorption, and excellent antimicrobial properties. It has received a lot of attention from researchers due to its many advantages $[8,9]$. Hydroxyapatite

whisker/nanoZnO-nmCaO (HAPw/nmZnO-nmCaO) composites were successfully prepared by the sol-gel method in the early stage of the group [10]. The ideal conditions for nanoparticle surface modification 
were derived from relevant experiments, i.e., the ideal temperature of $70^{\circ} \mathrm{C}$ for the reaction in a weak acid environment and other key modification steps. It was also concluded that $\mathrm{HAPw} / \mathrm{nmZnO}-\mathrm{nmCaO}$ composites possess good antibacterial and osteogenic properties. For hydroxyapatite whiskers (HAPw) have no porous structure, in this experiment, we intend to develop a coral hydroxyapatite/nanoZnO (CHA/nmZnO) composite with both porous, antibacterial and osteogenic properties using coral hydroxyapatite as the raw material. It is hoped to lay the foundation for the later investigation of the antibacterial and osteogenic properties of $\mathrm{CHA} / \mathrm{nmZnO}$ composites, and to provide relevant theoretical basis and experimental basis for the development of new antibacterial artificial bone repair materials.

\section{Materials and Methods}

\subsection{Experimental Materials}

Coral hydroxyapatite (CHA, homemade in the bioengineering materials laboratory of Kunming University of Science and Technology); diammonium hydrogen phosphate (analytical purity, Tianjin Fengchuan Chemical Reagent Technology Co., Ltd.); zinc nitrate (analytical purity, Sinopharm Reagent Co., Ltd.); polyethylene glycol-6000 (analytical purity, Sinopharm Chemical Reagent Co., Ltd.); anhydrous ethanol (analytical purity, Tianjin Fengchuan Chemical Reagent Technology Co.); ammonia (analytical purity, Tianjin Chemical Reagent Factory); glacial acetic acid (analytical purity, Chongqing Chemical Industry Group Co.).

\subsection{Instruments and Equipment}

S\&B Electronic balance (FA2004, Shanghai Haikang Electronic Instrument Factory); Ultrasonic cleaning machine (CQ250, No. 726, China Ship Seventh Hospital); Magnetic mixer (CJ78-1, Hangzhou Fuhua Instrument Co., LTD.); Constant temperature water bath (B-220, Shanghai Yarong Biochemical Instrument Factory); Rotary evaporator (RE-52CS, Shanghai Yarong Biochemical Instrument Factory); Digital display constant current pump (HL-BB, Shanghai Luxi Analytical Instrument Factory); box type resistance furnace (sRJX-1-2, Huanan Experimental Instrument Factory); digital display blast dryer (101A-1, Shanghai Experimental Instrument General Factory).

\subsection{Experimental Method}

\subsubsection{Preparation of $\mathrm{CHA} / \mathrm{nmZnO}$ Composites}

Natural coral was cleaned and disinfected and ground and sieved to obtain coral pellets, and CHA pellets were prepared according to the hydrothermal reaction principle using a special high-temperature and high-pressure reactor.

Zinc nitrate and polyethylene glycol-6000 (PEG-6000) were dissolved in $0.25 \mathrm{~L}$ anhydrous ethanol under water bath conditions at $70^{\circ} \mathrm{C}$ to obtain the clarified solution. The CHA pellets were placed in $0.25 \mathrm{~L}$ anhydrous ethanol and the clarified solution was added drop by drop into the CHA soaking solution under stirring conditions by means of a digital constant flow pump. The $\mathrm{pH}$ of the solution was adjusted to a weak acid environment using ammonia and glacial acetic acid, and ultrasonic dispersion was performed after the reaction in a constant temperature water bath at $70^{\circ} \mathrm{C}$. The temperature was continued to rise until the solvent was evaporated and dried, and subsequently placed in a drying oven for complete drying, and the resulting material was heat treated to obtain $\mathrm{CHA} / \mathrm{nmZnO}$ composites.

\subsubsection{Experimental Grouping}

The results of field emission scanning electron microscopy were used as test indicators, and the raw material ratios were gradually adjusted using a comprehensive experimental design to finally determine the raw material ratios, heat treatment temperatures, heat treatment holding times and other process conditions for the antimicrobial modification of CHA surfaces.

The samples were divided into a total of five groups according to the different ratios of raw material masses. The mass ratios of CHA, zinc nitrate and PEG-6000 were 4:2:1 (G1: group 1), 24:8:5 (G2: group 2), 24:4:5 (G3: group 3), 48:4:5 (G4: group 4) and 24:2:5 (G5: group 5).

According to the results of the simultaneous thermal analyzer, the composites were heat treated and the holding temperatures were set to $560^{\circ} \mathrm{C}, 580^{\circ} \mathrm{C}$ and $600^{\circ} \mathrm{C}$, respectively. The holding time was $1 \mathrm{~h}, 3 \mathrm{~h}$ and $5 \mathrm{~h}$, respectively, for a total of 9 groups.

\subsection{Test Methods}

\subsubsection{X-ray Diffractometer Detection (XRD)}

The samples under test were examined and analyzed by $\mathrm{X}$-ray diffractometer to investigate the change in the composition of the physical phase before and after the modification of the samples.

\subsubsection{Scanning Electron Microscope Inspection (SEM)}

The CHA/nmZnO composites were examined using SEM. The surface morphology, pore size, nanoparticle size, distribution and other structural features were analyzed.

\subsubsection{Electron Energy Spectroscopy (EDX)}

The elemental classes and relative contents of the samples in the specified area were analyzed using X-ray photoelectron spectrometry (K-Alpha+, Thermo Fisher Scientific Ltd.) under SEM detection.

\subsubsection{Synchronous Thermal Analyzer Assay (TGA)}

The results of thermogravimetric analysis were used to study the decomposition temperature and other characteristics of the composite, and to investigate the key process conditions such as holding temperature and holding time during heat treatment of the composite.

\section{Results}

\subsection{XRD Test Results}

The data related to the samples were obtained by XRD 
detection, and the elemental composition of the material under test was analyzed using Jade 6.5 software. The analysis results were graphically plotted by Origin 8.0 and the XRD profiles of the samples were obtained as shown in Figure 1.

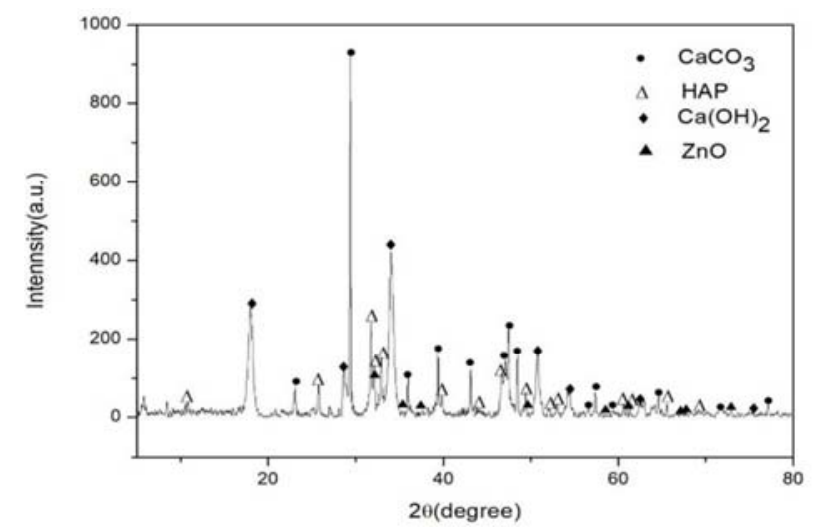

Figure 1. XRD analysis results.

From the XRD test pattern it can be concluded that: The composites are mainly composed of calcium carbonate. The characteristic peak of calcium hydroxide may be due to the denaturation of some calcium carbonate to form calcium oxide after heat treatment of the composites, which chemically reacts with the moisture in the air. Also the presence of HAP characteristic peak in the sample indicates that the surface coral carbonate component was converted to hydroxyapatite after hydrothermal reaction of natural coral. The presence of $\mathrm{ZnO}$ characteristic peak in the sample also proves that the composite obtained by surface modification of CHA using sol-gel method contains $\mathrm{ZnO}$ component.

\subsection{SEM Test Results}

The SEM results of each group of materials are shown in Figure 2. According to the SEM results, it can be seen that the $\mathrm{nmZnO}$ particles on the surface of the composite in G4 (group IV) are uniformly distributed with ideal particle size, the porous structure of the material is not destroyed, and the nanoparticle fusion attachment is ideal.

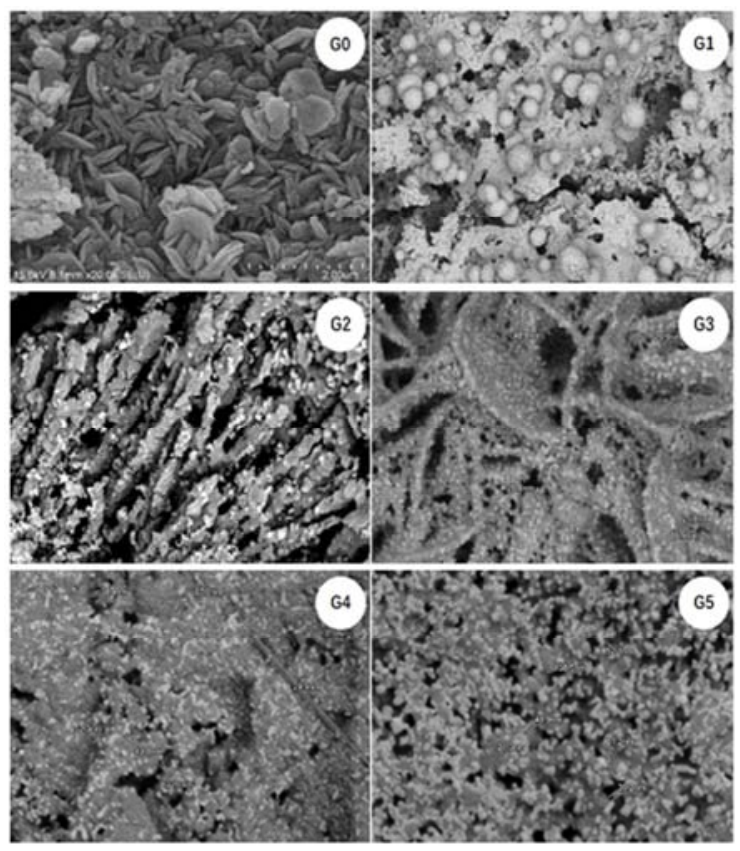

Figure 2. SEM images of G0-G5 group.

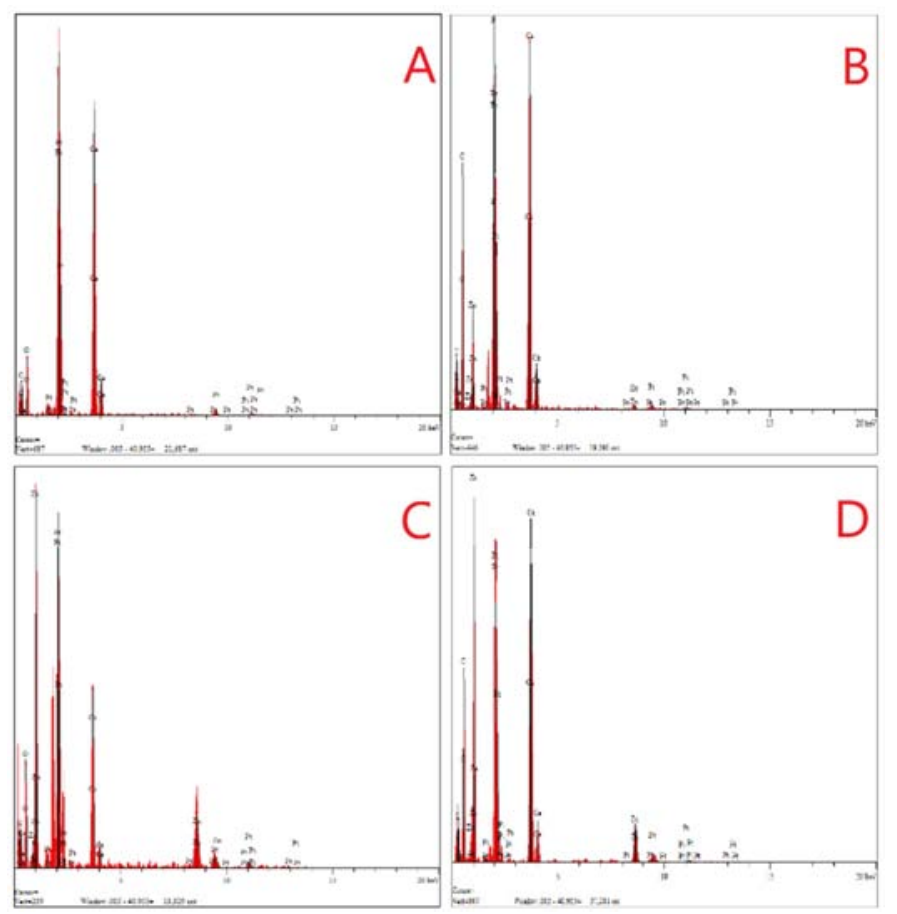

3a: EDX test results of group G0 (CHA control group); 3b: EDX test results of group G3; 3c: EDX test results of group G4; 3d: EDX test results of group G5

Figure 3. EDX analysis results. 


\subsection{EDX Assay Results}

The EDX test results of the control group and the G3-G5 group are shown in Figure 3. The EDX test results indicate that the $\mathrm{CHA} / \mathrm{nmZnO}$ composite is composed of five elements, $\mathrm{Zn}, \mathrm{Ca}, \mathrm{O}, \mathrm{P}$, and $\mathrm{C}$. The results confirm that the nanoparticles on the surface of the composite are $\mathrm{nmZnO}$ particles. The presence of Pt elements may be due to the material surface after platinum spray treatment.

\subsection{TGA Test Results}

The heat loss curves (TG curves), differential scanning calorimetry curves (DSC curves) and heat loss differential curves (DTG curves) of the test samples were obtained after the CHA pellet samples were warmed up to $900^{\circ} \mathrm{C}$ at a heating rate of $10^{\circ} \mathrm{C} / \mathrm{min}$ in an air atmosphere as shown in Figure 4.

Table 1. Changes of composites after heat treatment.

\begin{tabular}{llll}
\hline $\begin{array}{l}\text { Insulation } \\
\text { temperature }\left({ }^{\circ} \mathbf{C}\right)\end{array}$ & Keep warm for $\mathbf{1 h}$ & Keep warm for $\mathbf{3 h}$ & Keep warm for $\mathbf{5 h}$ \\
\hline 560 & $\begin{array}{l}\text { Black color, the original structure is not } \\
\text { destroyed } \\
\text { Color gray, the original structure is not } \\
\text { destroyed } \\
\text { Grayish white color, the original } \\
\text { structure is not destroyed }\end{array}$ & $\begin{array}{l}\text { Grayish Black color, the original structure } \\
\text { is not destroyed } \\
\text { Grayish white color, the original structure } \\
\text { is not destroyed } \\
\text { White yellow color, the original structure } \\
\text { is not destroyed }\end{array}$ & $\begin{array}{l}\text { Grayish white color, the original structure } \\
\text { is not destroyed } \\
\text { White color, the original structure is not }\end{array}$ \\
\hline
\end{tabular}

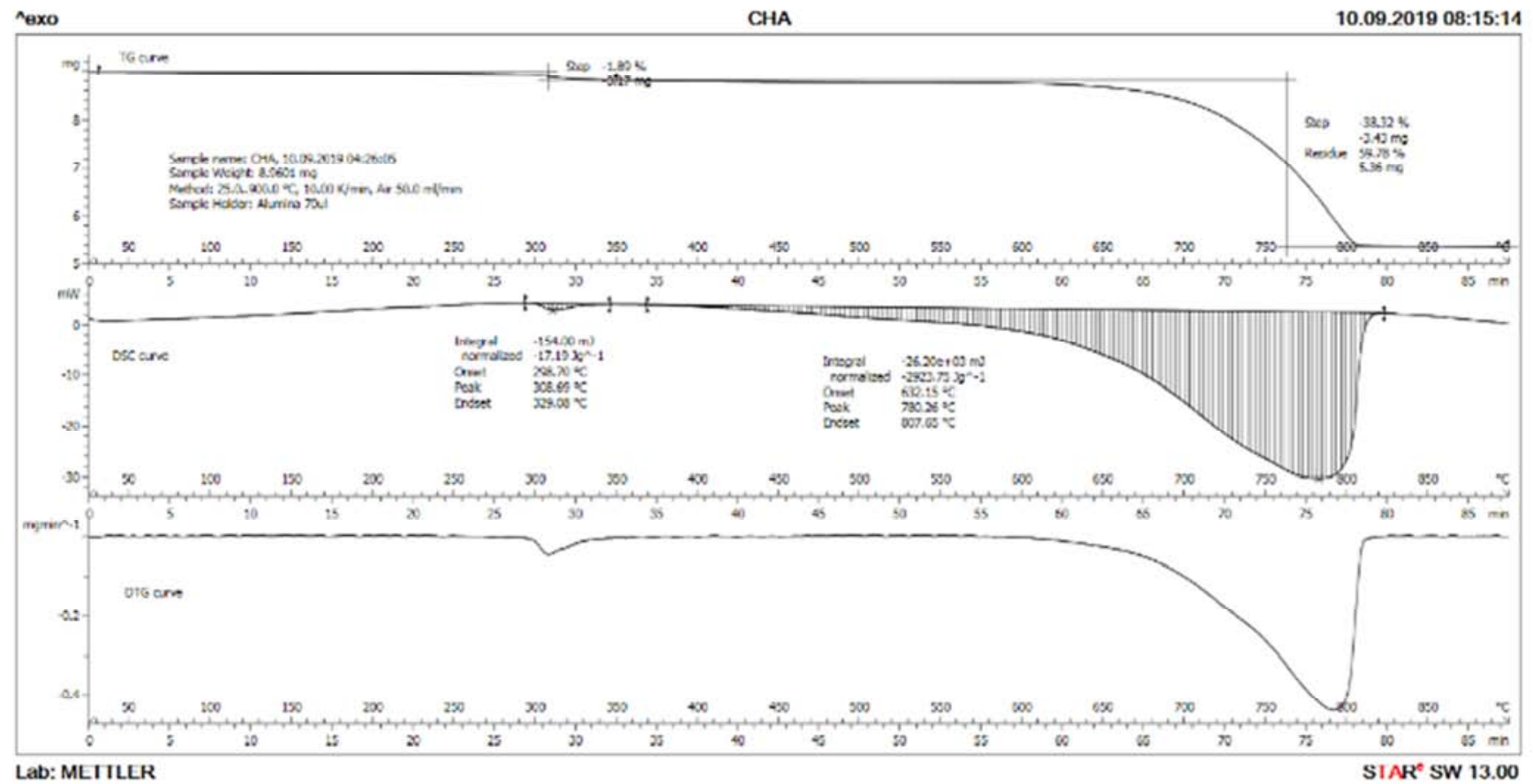

Figure 4. TGA analysis results of the CHA.

From the TG curve, it can be concluded that CHA particles start to produce weight change at $632.15^{\circ} \mathrm{C}$. The most significant weight loss of the sample was observed at $780.26^{\circ} \mathrm{C}$. The weight loss reaction process was completed at $807.65^{\circ} \mathrm{C}$ with a total weight loss of $40.22 \%$. The DTG curve results were in agreement with the TG curve results. It was concluded that the heat treatment temperature of the composites should be controlled below $632.15^{\circ} \mathrm{C}$ for heat treatment.

\subsection{Results After Heat Treatment of $\mathrm{CHA} / \mathrm{nmZnO}$}

The mixed gel material was subjected to heat treatment experiments, and the working procedure of the resistance furnace was set: the temperature rise rate was $2^{\circ} \mathrm{C} / \mathrm{min}$. The holding temperature and holding time were set according to the experimental design, and the surface color and texture of the material were generally observed after heat treatment as shown in Table 1.

According to the experimental results, it is known that. With the increase of holding temperature and holding time, the more ideal the decarburization effect is, but the worse the strength of the material is, which eventually leads to the destruction of the porous structure of the material. The optimal heat treatment conditions for $\mathrm{CHA} / \mathrm{nmZnO}$ composites are as follows: The temperature increase rate is $2^{\circ} \mathrm{C} / \mathrm{min}$ to $580^{\circ} \mathrm{C}$, and the holding time is $5 \mathrm{~h}$, the decarburization effect and mechanical strength of the 
material are more ideal, and the original structure is not damaged.

\section{Discussion}

In the traditional treatment of infected bone defects, debridement combined with antibiotics is usually used for treatment. However, due to poor blood supply conditions at the trauma site, it is difficult to maintain an effective antibacterial concentration at the infected site, such as oral or intravenous antibiotics [11], and the post-healing effect is often poor. Currently, common antimicrobial bone repair materials are loaded with antibiotics on artificial bone materials, thus possessing antimicrobial effects [12, 13]. However, superbugs have also been reported more frequently in recent years [14], thus limiting the development of this material. There are many reasons for the formation of drug-resistant strains [15]: for example, misuse of antibiotics and improper combination of drugs in clinical work [16], mutation of bacteria themselves, biofilm formation by proteins secreted by bacteria, the influence of drug-resistant plasmids and the exocytosis of bacteria [17]. The advantages of inorganic antibacterial agents are more obvious compared to antibiotics. Inorganic antibacterial agents are safe, have broad-spectrum and long-lasting antimicrobial effects, and do not produce drug resistance. Inorganic antimicrobial agents are generally photocatalytic semiconductor antimicrobial materials and antimicrobial active metals and their oxides [18], the former limit their application due to photocatalytic conditions, so the latter have received extensive attention from researchers. Among them, $\mathrm{ZnO}$ nanoparticles have abundant raw material sources, lower prices, non-toxic to the ecological environment, and their broad forbidden bands, UV absorption, and excellent antimicrobial properties have attracted much attention in the field of antibacterial materials.

Coral hydroxyapatite is a composite material in which the surface calcium carbonate is replaced with hydroxyapatite by a "hydrothermal reaction" of natural coral [5]. This material not only has good biocompatibility and osteoconductivity of hydroxyapatite, but also enhances the mechanical strength of natural coral and retains the original porous structure of coral. This property facilitates the growth of new bone tissue. It has been well documented that coral hydroxyapatite contributes to bone and soft tissue ingrowth and coral endosteal reconstruction [19-20]. The degradation rate can also be artificially controlled by adjusting the "hydrothermal reaction" conditions and changing the hydroxyapatite conversion rate [21]. In recent years, $\mathrm{CHA}$ artificial bone has been widely used in the treatment of oral and maxillofacial bone defects [22].

The conclusions of the key steps of this experiment are consistent with the previous results of the group [23]. The resulting $\mathrm{CHA} / \mathrm{nmZnO}$ composite artificial bone material is a new antibacterial biomaterial combining coral hydroxyapatite with porous structure and good biocompatibility with nanoscale inorganic metal oxides. This makes CHA artificial bone material with antibacterial effect. It has been shown that $\mathrm{ZnO}$ nanoparticles have long-lasting and broad-spectrum antimicrobial properties [24] and are a hot spot for research among inorganic antimicrobial materials. Also the calcium ions released during the degradation of $\mathrm{CHA}$ artificial bone materials are beneficial to the growth and proliferation of osteoblasts [25, 26]. The phosphate ions in CHA degradation products not only facilitate biomineralization, but also are a signaling molecule for osteoblast differentiation protein production and gene expression [27]. The next step of the group will be to conduct experimental investigations related to the antibacterial and osteogenic properties of $\mathrm{CHA} / \mathrm{nmZnO}$ composites.

\section{Conclusion}

The following conclusions can be drawn from the various experimental conditions of surface modification of coral bone hydroxyapatite using the sol-gel method: (1) The surface modification of CHA particles can be performed using the sol-gel method, and uniformly distributed spherical $\mathrm{ZnO}$ nanoparticles can be observed under scanning electron microscopy. (2) The optimal conditions for the modification of CHA particles using the sol-gel method are: The $\mathrm{pH}$ is to be adjusted to a weak acid condition, the reaction temperature is $70^{\circ} \mathrm{C}$, and the mass ratio of CHA, zinc nitrate and PEG-6000 is 48:4:5. (3) The ideal process condition for heat treatment of the material is to set the holding temperature at $580^{\circ} \mathrm{C}$ and holding time at $5 \mathrm{~h}$ under the condition of uniform temperature increase at $2^{\circ} \mathrm{C} / \mathrm{min}$.

\section{References}

[1] Xu Ling, Lv Kaige, Zhang Wenjie, et al. The healing of critical-size calvarial bone defects in rat with rhPDGF-BB, BMSCs, and $\beta$-TCP scaffolds [J]. Journal of materials science, 2012, 23 (4): 1073-1084.

[2] Guo Jun, Meng Zhaosong, Chen Gang, et al. Restoration of critical-size defects in the rabbit mandible using porous nanohydroxyapatite-polyamide scaffolds [J]. Tissue engineering, Part A, 2012, 18 (11-12): 1239-1252.

[3] Rentsch Claudia, Rentsch Barbe, Breier Annette, et al. Long-bone critical-size defects treated with tissue-engineered polycaprolactone-co-lactide scaffolds: a pilot study on rats [J]. Journal of biomedical materials research, Part A, 2010, 95 (3): 964-972.

[4] N. Mokbel, C. Bou Serhal, G. Matni, et al. Healing patterns of critical size bony defects in rat following bone graft $[\mathrm{J}]$. Oral and maxillofacial surgery, 2008, 12 (2): 73-78.

[5] Roy D M, Linnehan S K. Hydroxyapatite formed from coral skeletal carbonate by hydrothermal exchange [J]. Nature, 1974, Vol. 247 (5438): 220-222.

[6] Dodds RA, York - Ely AM, Zhukauskas R, et al. Biomechanical and radiographic comparison of demineralized bone matrix, and a coralline hydroxyapatite in a rabbit spinal fusion model [J]. J Biomater Appl. 2010, 25 (3): 195-215. 
[7] Wasielewski RC, Sheridan KC, Lubbers MA. Coraline hydroxyapatite in complex acetabular reconstruction [J]. Orthopedics, 2008, 31 (4): 367.

[8] Huo K, Zhang X, Wang $H$. Osteogenic activity and antibacterial effects on titanium surfaces modified with $\mathrm{zn}$-incorporated nanotube arrays [J]. Biomaterials, 2013; 34 (13): 3467-3478.

[9] Kasraei S, Sami L, Hendi S. Antibacterial properties of composite resins incorporating silver and zinc oxide nanoparticles on streptococcus mutans and lactobacillus [J]. Restor dent endod, 2014; 39 (2): 109-114.

[10] Sun Shijia. Preparation and performance study of $\mathrm{HAPw} / \mathrm{nmZnO}-\mathrm{nmCaO}$ antibacterial bone repair materials [D]. Kunming: Master's thesis, Kunming Medical University, 2017.

[11] Malat TA, Glombitza M, Dahmen J, et al. The use of bioactive glass S53P4 as bone graft substitute in the treatment of chronic osteomyelitis and infected non-unions - a retrospective study of 50 patients [J]. Z Orthop unfall, 2018, Vol. 156 (2): 152-159.

[12] Tao Cheng, Haiyun Qu, Guoyou Zhang, et al. Osteogenic and antibacterial properties of vancomycin-laden mesoporous bioglass/PLGA composite scaffolds for bone regeneration in infected bone defects [J]. Artificial cells, nanomedicine, and biotechnology, 2018, Vol. 46 (8): 1935-1947.

[13] Wei S, Jian $\mathrm{C}$, Xu F, et al. Vancomycin-impregnated electrospun polycaprolactone (PCL) membrane for the treatment of infected bone defects: An animal study $[\mathrm{J}]$. J Biomater Appl, 2018, 32 (9): 1187-1196.

[14] Liu D-H, Zhang X-W, Zhang C. Antibiotic abuse and superbugs [J]. Foreign Medicine (Antibiotics Branch), 2019, $40(1): 1-4$

[15] Xie X. L., Bai F. Scientists reveal the molecular mechanism of bacterial drug resistance generation [J]. Microbiology Bulletin, 2016, 43 (5): 1164

[16] Lindner T, Kanakaris NK, Marx B, et al. Fractures of the hip and osteoporosis: the role of bone substitutes [J]. Bone Joint Surg Br, 2009, 91 (3): 294-303.

[17] Ji H-B. Analysis of bacterial resistance to antibacterial drugs [J]. Chinese and Foreign Medical Care, 2018, 37 (2): 92-195.
[18] Xiang R, Ding D-B, Fan L-L, et al. Progress of antibacterial mechanism of zinc oxide and its safety research $[\mathrm{J}]$. Chinese Tissue Engineering Research, 2014, 18 (3): 470-475.

[19] Fu Kun, Xu Qingguo, Czernuszka Jan, et al. Characterization of a biodegradable coralline hydroxyapatite/calcium carbonate composite and its clinical implementation [J]. Biomedical materials, 2013, 8 (6): 1327-1329.

[20] Chen Zepeng, Zhang Ying, Lin Zefeng, et al. Imaging evaluation of dextran sulfate/recombinant human BMP-2/chitosan composite microspheres combined with coral hydroxyapatite artificial bone for repairing large segmental bone defects [J]. Chinese Journal of Restorative and Reconstructive Surgery, 2017, 31 (11): 1384-1389.

[21] Liu Chang. Study on in vitro degradation of coral hydroxyapatite with different replacement rates [D]. Zhengzhou: Master's thesis, Zhengzhou University, 2016.

[22] Hak DJ. The use of osteoconductive bone graft substitutes in orthopaedic trauma $[\mathrm{J}]$. The journal of the american academy of orthopaedic surgeons, 2007, Vol. 15 (9): 525-536.

[23] Sun Shijia, Zhao Qiang, Yuan Yanbo, et al. Study on the effect of $\mathrm{pH}$ on the morphology of HAPw-nmZnO-nmCaO composite biomaterials [J]. Journal of Dental Research, 2017, 33 (6): 585-588.

[24] Zeng Xianli. Study on the antifungal mechanism of zinc oxide nanoparticles [D]. Zhuzhou: Master's thesis, Hunan University of Technology, 2017.

[25] Mladenovic Z, Sahlin-Platt A, Andersson B, et al. In vitro study of the biological interface of bio-oss: implications of the experimental setup [J]. Clinical oral implants research, 2013, Vol. 24 (3): 329-335.

[26] El-Ghannam A, Ning C Q. Effect of bioactive ceramic dissolution on the mechanism of bone mineralization and guided tissue growth in vitro [J]. Journal of biomedical materials research. Part A, 2006, Vol. 76 (2): 386-397.

[27] Beck George R. Inorganic phosphate as a signaling molecule in osteoblast differentiation [J]. Journal of cellular biochemistry, 2003, 90 (2): 234-243. 